\title{
Image processing and transmission scheme based on generalized Gaussian mixture with opportunistic networking for wireless sensor networks
}

\author{
Jingbin HaO
}

\begin{abstract}
For satisfying the quality of service (QoS) requirements and image denoising services in wireless sensor network (WSN) applications, based on opportunistic networking technology and generalized Gaussian mixture algorithm, an adaptive image processing and transmission scheme is proposed in this paper. According to the real-time state record matrix, the multi-objective optimization scheme with equalizer coefficients and the opportunistic cooperative scheme in view of energy and computing ability are studied, respectively. Then, the generalized Gaussian mixture algorithm is used to reduce the image data and eliminate the noise interference from the WSN environment. Finally, Simulation results show that the proposed scheme has better QoS support capability results such as reliability, real-time performance, and energy efficiency, as well as the image decoding accuracy including peak signal to noise ratio.
\end{abstract}

\section{Introduction}

With the development of wireless communication technology, mobile technology, and decline of sensor hardware cost, image capture, and transmission, video communication has been widely developed in the wireless sensor network (WSN) applications [1], which include the traffic detection, license plate recognition, object tracking and location applications, [2] etc. However, the energy, bandwidth and processing power of sensor, is limited and WSNs have the characteristics such as dynamic topology and instability and resource-constrained systems. WSNs have the characteristics such as dynamic topology, instability and resource-constrained systems, etc. Specially, with development of opportunistic network technology, how to conduct the following operations: image or video capture, wireless transmission, encoding and decoding, storage and so on, brought a series of challenges and opportunities.

For reducing communication overheads, based on highresolution captures, the higher data reduction rates can be used in WSNs by removing redundant parts of the image

Correspondence: jbinhao@163.com

College of Mechanical and Electrical Engineering, China University of Mining and Technology, Xuzhou 221116, China

\section{Springer}

capture [3]. Two image transmission schemes were proposed according to energy efficiency considerations which are open-loop and closed-loop image transmission schemes [4]. Based on the open stationary APs and spontaneous mobile APs, the WiFi-Opp algorithm was proposed in [5], which is a realistic opportunistic setup and enable opportunistic communications. In article [6], the authors proposed a novel technique with collaborative image transmission in WSNs for improving the energy efficiency in period of redundant data transmission.

However, the above researches are not applicable for WSNs because of their hardware development complexity, energy consumption of image processing, and resource utilization of image communication. In addition, how to process, compress, and transport the image or video with opportunistic networking in WSNs has been ignored.

In this paper, based on opportunistic networking and computing, we focus on the robust and efficient image processing and transmission scheme with generalized Gaussian mixture model to satisfy the service requirements of image or video application in resource constraint WSNs and extend the life cycle of WSNs. 
The rest of the paper is organized as follows. The related work is given in Section 2. Image transmission based on opportunistic networking for WSNs is studied in Section 3. In Section 4, the image processing scheme based on generalized Gaussian mixture model was designed in WSNs. The adaptive image processing and transmission scheme based on generalized Gaussian mixture modeling with opportunistic networking is proposed and used to provide the robust and effective image communication in WSNs, which is shown in Section 5. Experiment results are given in Section 6. Finally, we conclude the paper in Section 7.

\section{Related work}

The image processing algorithm for WSNs has made the following findings. Some compression algorithms have been designed for WSNs [7], which include coding algorithm by ordering, pipelined in-network compression, low-complexity video compression, and distributed compression. To decrease the hardware cost and energy consumption, $\mathrm{Lu}$ et al. [8] proposed the lowcomplexity and energy efficient image compression scheme, which reduced the computational complexity and required memory. A hardware solution for userdriven and packet loss tolerant image compression was presented and evaluated [9], which was designed to enable low power image compression and communication over wireless camera sensor networks. The control architecture was proposed by George Nikolakopoulos et al. [10] according to the quality of the transmitted images with the traffic load within the network and the level of details contained in an image frame. There are some researchers study the algorithm to reduce the energy consumption in WSNs. It is well known that the data reduction scheme of energy conservation could be used to lower the power consumption. Specially, S. K. Soni et al. [11] implemented the data reduction scheme by the prediction approach based on $\operatorname{GM}(1,1)$ model. According to the importance and priority of data blocks, Kerem Irgan et al. [12] researched a simple encoding scheme at the source sensor. The WSNs would transmit the important or high priority data blocks with reliable paths.

At the same time, the novel architecture and protocol for energy efficient image processing and communication over WSNs was presented by author of [13], which could make image communication over WSNs feasible, reliable, and efficient. To address the limitation of restricted computational power, memory limitations, narrow bandwidth, and energy supplied in sensor nodes, M. Nasri et al. [14] studied an image transmission approach in WSNs. On the basis of sensing relevancies, an energy efficient visual monitoring mechanism was presented by each source sensor [15], which would be a function of the monitoring relevance of the node for the application. Contrary to the lack of processing capability and the queue control and error detection capabilities, Duc Minh Pham et al. [16] proposed an innovative architecture for object extraction and a robust application-layer protocol for energy efficient image communication over WSNs. In view of resource constraints and challenge of digital image transmission for image-sensor-based WSNs, the design and implementation of WSNs with low costs, low power, and based on a low rate ZigBee protocol was presented and evaluated in [17]. When transmitting still images on a multi-hop network, the performance level would be determined by identifying limitations and bottlenecks of sensor board hardware and 802.15.4 radio [18].

On the other hand, opportunistic networking technology has been one of the host issues in WSNs, in which the mobile sensor would communicate with each other even if a route between them never exists. In article [19], the authors survey the most interesting cases with opportunistic networking and discuss a taxonomy for the main routing and forwarding approaches. In the light of mobility, social relations, and communication, Theus Hossmanna et al. [20] analyzed two datasets comprising social, mobility, and communication ties. To address the problem how to create and maintain the opportunistic networks, the probabilities of the establishment and maintaining were analyzed by J. Gebert et al. [21] in the different scenarios. The evolution from opportunistic networking to opportunistic computing was researched in [22]. In addition, author of [22] surveyed the key recent achievements in opportunistic networking and described the main concepts and challenges of opportunistic computing. Against to the transmissions of large sized images and security, a collaborative transmission scheme for image sensors was proposed in [23] to utilize inter-sensor correlations based on the path diversities. On the basis of the combination of wavelet-based image decomposition and cooperative communication, an energy efficient image transmission strategy for WSNs was proposed [24]. A picture delivery service for camera sensor networks was proposed in [25], which is motivated by the needs of disaster-response applications. Zhi Ren et al. [26] studied an efficient low-delay routing algorithm, epidemic routing according to the adaptive compression of vectors, which could adaptively reduce the length of SVs and RVs by compressing the same bit sequences. Due to compressed sensing, the novel signal separation algorithm was studied and presented for recovering the entire spectrum and separating mixed occupying signals in [27]. In article [28], the dimensionality reduction targeting the preservation of multimodal structures was proposed to counter this parameterspace issue. 


\section{Image transmission based on opportunistic networking}

According to the topology shown in Fig. 1, the WSNs were deployed. Each sensor node is configured some image acquisition device such as a camera or video camera, which five would be deployed in the monitoring area according to the monitoring requirements. Sensor nodes can monitor the acquisition of image data and forward image or video sequence to an aggregation node with cooperative transmission.

The figure shows a heterogeneous WSN, which includes the sink node, sensor node with the video camera or with the camera. Captured images or video information would be transmitted through mutual cooperation between heterogeneous sensor nodes to sink node. The image data would be processed and forwarded to the server storage. Users could obtain the image data through a wireless or wired link to access server. They also could get the data via mobile phone and data processing could be operated on the phone.

In order to improve link utilization rate and system resources, each sensor node would calculate the remaining energy capacity by sensing and obtain the statistical quality of the link, then opportunistically access network for transmission of image information.

The heterogeneous WSN topology is defined as an undirected graph, which is composed by the two collections $V$ and $E$ and denoted by $G=(V, E)$. Here, $V$ is a finite nonempty set of heterogeneous sensor nodes. $E$ is the set of the radio link between the sensors of $V$ and the wireless link is a nondirectional. Let $\phi=\left\{E_{R}, A_{c}\right\}$ real-time status of each node with vector. Let $E_{R}$ denote the remaining energy. Let $A_{c}$ denote the computing ability. $\Phi=\left[\phi_{1}, \cdots \phi_{N}\right]$ is defined to record real-time information about all network sensors.

Based on current real-time network status and information of sensors, considering every time image transmission with a clear transmission path, undirected graph $G$ will be converted into a directed graph $\bar{G}$. Figure 2 gives an undirected graph $G_{1}=\left(V_{1}, E_{1}\right)$. Here, $V_{1}=\left\{V_{A}, V_{B}, V_{c}, V_{D}\right.$, $\left.V_{E}\right\}$ and $E_{1}=\left\{\left\langle V_{A}, V_{B}\right\rangle,\left\langle V_{B}, V_{c}\right\rangle,\left\langle V_{c}, V_{D}\right\rangle,\left\langle V_{D}, V_{A}\right\rangle,\left\langle V_{D}, V_{B}\right\rangle\right.$, $\left.\left\langle V_{A}, V_{E}\right\rangle,\left\langle V_{E}, V_{c}\right\rangle\right\}$. When the wireless link of image data transmission is $V_{A} \rightarrow V_{B}, V_{B} \rightarrow V_{E}, V_{C} \rightarrow V_{D}, V_{D} \rightarrow V_{E}$, $V_{B} \rightarrow V_{E}, V_{A} \rightarrow V_{E}$, and $V_{E} \rightarrow V_{C}$, network topology is shown as Fig. 3. That is, a directed graph $\bar{G}_{1}=$ $\left\{\bar{V}_{1}, \bar{E}_{1}\right\}$. Here, $\bar{V}_{1}=\left\{V_{A}, V_{B}, V_{C}, V_{D}, V_{E},\right\}$ and $\bar{E} 1=\left\{\left(V_{A}, V_{B}\right),\left(V_{B}, V_{E}\right),\left(V_{c}, V_{D}\right),\left(V_{D}, V_{E}\right),\left(V_{B}, V_{E}\right)\right.$, $\left.\left(V_{A}, V_{E}\right),\left(V_{E}, V_{c}\right)\right\}$. The optimal transmission routing path could be obtained by transfer form Figs. 2 and 3.

Due to the randomness of the channel, dynamic topology and resource-constrained WSNs, all information is sent to the sink node with multiple routing transmission. That means, the image data would be able to be sent on all possible link. Undirected graph will be converted into a directed graph and then stored in the relay nodes, to facilitate end to end communication.

Each node in WSNs must satisfy the principle of proportionality resources to join the opportunity communication. Remaining energy resources and computational power of the image data reception node is equal to or

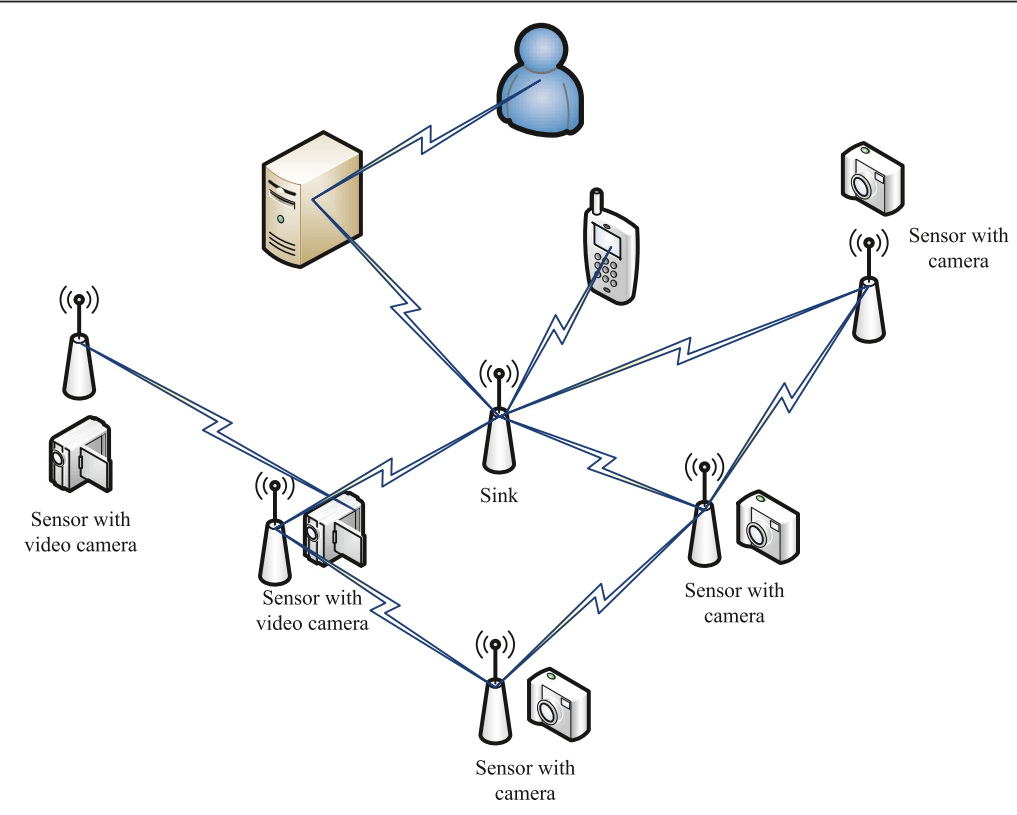

Fig. 1 Topology of WSNs with camera or video camera 


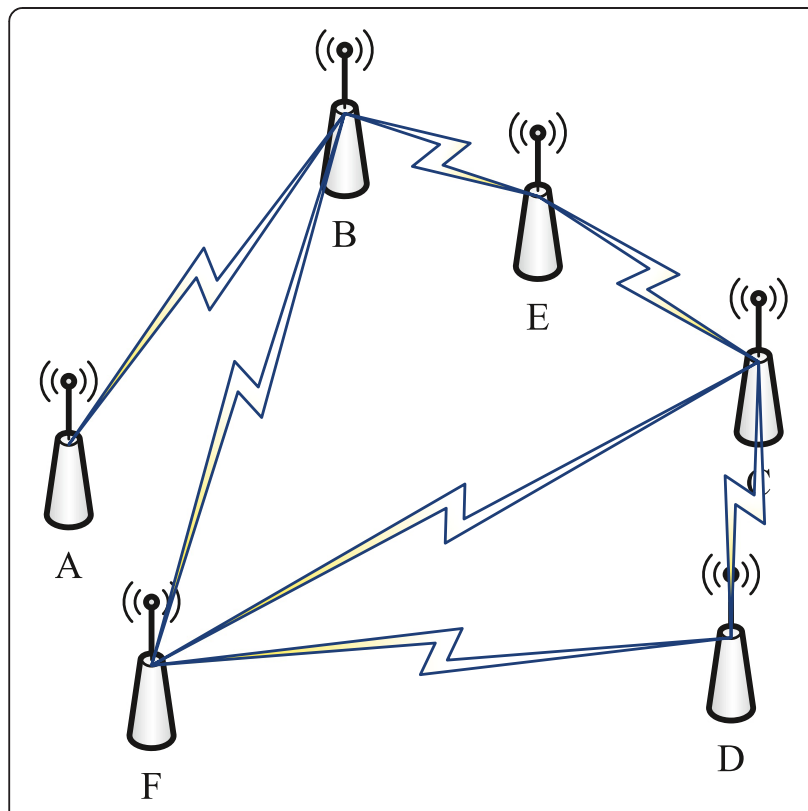

Fig. 2 Undirected graph $G_{1}$

greater than the image data transmission node in order to opportunistic data forwarding, which is shown as formula (1).

$$
\phi_{R} \geq \phi_{S} \quad \text { i.e., } \quad\left\{\begin{array}{l}
E_{R}(R) \geq E_{R}(S) \\
A_{c}(R) \geq A_{c}(S)
\end{array}\right.
$$

Here, let $\phi_{R}$ and $\phi_{S}$ denote the real-time status of image data transmitting and receiving or forwarding node, respectively. Let $E_{R}(R)$ and $E_{R}(S)$ denote the

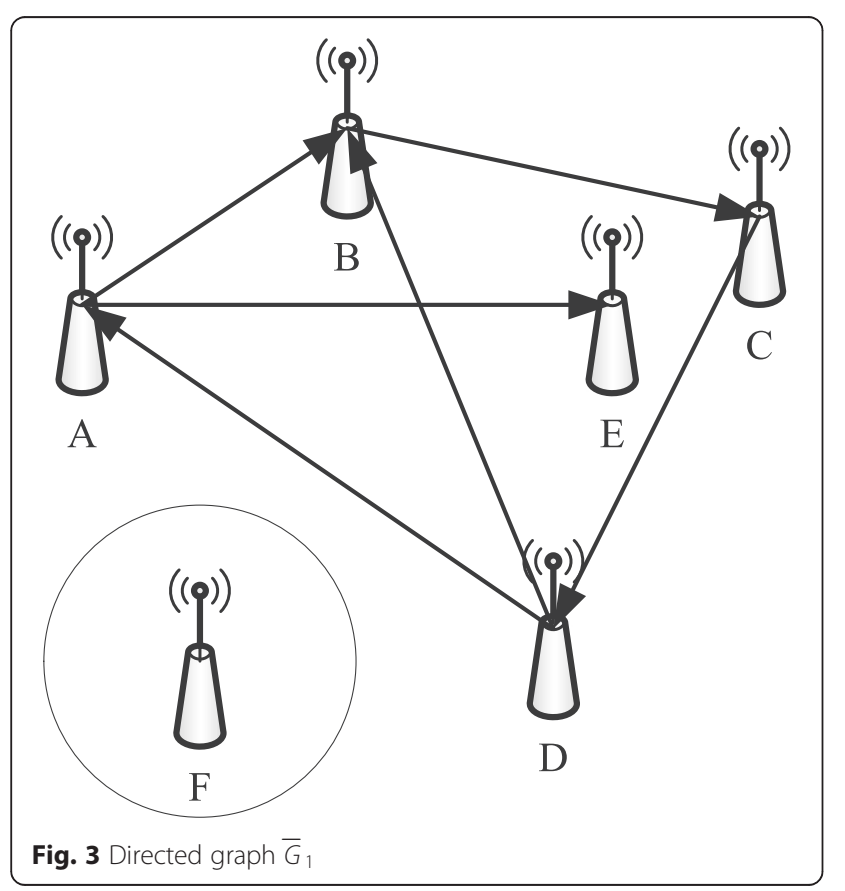

remaining energy of image data transmitting and receiving or forwarding node, respectively. Let $A_{c}(R)$ and $A_{c}(S)$ denote the computing ability of image data transmitting and receiving or forwarding node, respectively.

In view of randomness in the physical layer of wireless links, limited resources, and the conditions under multipath routing, the target of image transmission in WSNs based on the opportunistic networking is to ensure the image information transmission quality while maintaining minimal transmission delay, energy efficient. However, the above three design goals are mutually contradictory and not simultaneously satisfied in practical applications. It is necessary the compromise image data transmission scheme. The tradeoff between three objectives could be optimized by introducing weights $\mu=\left[\mu_{1}, \cdots \mu_{N}\right]^{T}$.

$$
\left\{\begin{array}{cc}
\text { maximize } & \Phi \cdot \mu \\
\text { subject to } & \phi_{R} \geq \phi_{S}
\end{array}\right.
$$

Image transmission algorithm of WSNs with opportunistic networking is described as follows:

(1)Determine the optimal weights $\mu$ based on requirement of WSNs image applications.

(2) Set the matrix $\Phi$ of real-time state vector in each node through the self-organizing properties of WSNs, which save the corresponding node residual energy and computing power to identify.

(3) According to the network topology, undirected graph $G$ is set to determine link conveniently.

The above three steps of initialization phase are used to reduce the resource consumption and energy consumption, as well as the complexity of the algorithm and hardware complexity.

(4) The sending sensor of image data broadcasts the threshold $s=\left\{E_{R}(S), A_{c}(S)\right\}$ of residual energy and computing power to neighbor sensors.

(5) According to the formula (1), neighbor sensor nodes determine whether to join the cooperative communication.

(6) After operating of step (5) at all neighbor sensor, a matrix of opportunities communication sensor nodes would be formed. According to the formula (2) and (3), objective optimization would be resolved and get the opportunistic networking path.

(7) The image routing path is converted into the directed graph digraph $\bar{G}$.

(8) When the transmission path is interrupted, execution of step (4)-(7) is to satisfy the opportunity to optimization target image transmission and reestablish the communication path. 


\section{Image processing based on generalized gaussian mixture model}

Before image transmission in WSNs, the images with noisy could be denoted by the following matrix.

$$
\left[\begin{array}{c}
y_{1} \\
\vdots \\
y_{M}
\end{array}\right]=\left[\begin{array}{c}
x_{1} \\
\vdots \\
x_{M}
\end{array}\right]+\left[\begin{array}{c}
N \\
\vdots \\
N
\end{array}\right]
$$

where let matrix $\left[y_{1}, \ldots ., y_{M}\right]^{T}$ denotes the image with monitored noisy, let matrix $\left[x_{1}, \cdots, x_{M}\right]^{T}$ denotes the image with no noise interference. The matrix $[N, \cdots, N]^{T}$ represents the Gaussian white noise with zero mean. Additionally, $x_{i}(\mathrm{i}=1,2, \ldots, M)$ could be calculated by the formula (4).

$$
p\left(x_{i} \mid \mu_{i}\right)=\sum_{l=i}^{U} \delta_{k}^{X} G\left(\bar{X} \mid \mu_{l}^{\bar{X}}\right)(i=1,2, \ldots, M)
$$

where $\mu_{1}^{\bar{X}}, \mu_{2}^{\bar{X}}, \cdots, \mu_{i}^{\bar{X}}$ represent the coefficient vector of objective optimization. We can initiate the vector according to the real measurement of the sensor system. Let $\sum_{k=i}^{K} \delta_{k}(i=1,2, \ldots, M)$ denotes the generalized mixing coefficient, sum of which must be 1 . Let $\bar{X}$ denotes the sum of $x_{i}(\mathrm{i}=1,2, \ldots, M)$. So, the function of $G$ $\left(\bar{X} \mid \mu_{l} \bar{X}\right)$ denotes generalized distribution process. Density function $p\left(x_{i} \mid \mu_{i}\right)[29,30]$ represents the mixing process.

Figure 4 found that, the maximum coefficient value generalized distribution density function is always increasing with the increase of $\mu$, which is set to different values that indicate that it is necessary to select the optimum weight based on the actual requirement of image processing.

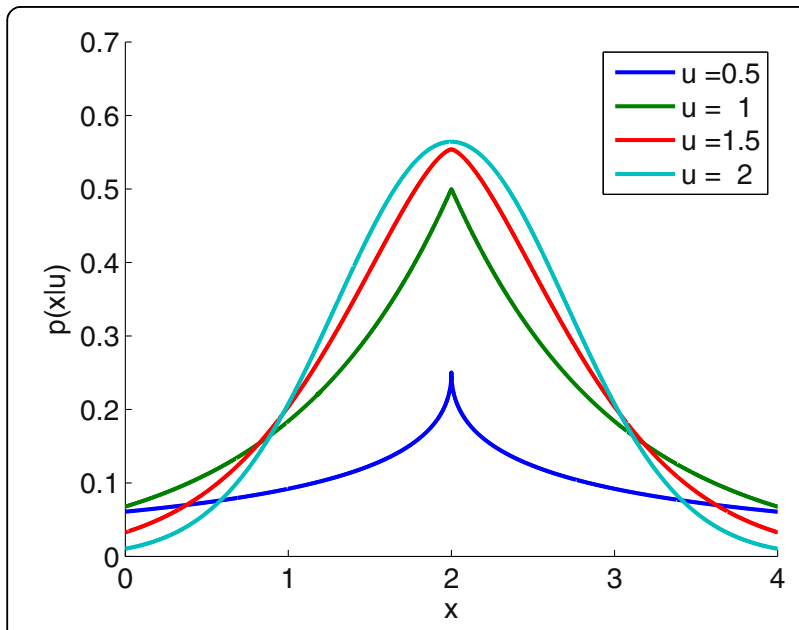

Fig. 4 Density with $\mu$
Figure 5 shows the generalized Gaussian distribution density function based on three different optimization objectives. When the image data is processed with the Gaussian mixed model, after considering the remaining energy, delay, and computing ability, the distribution

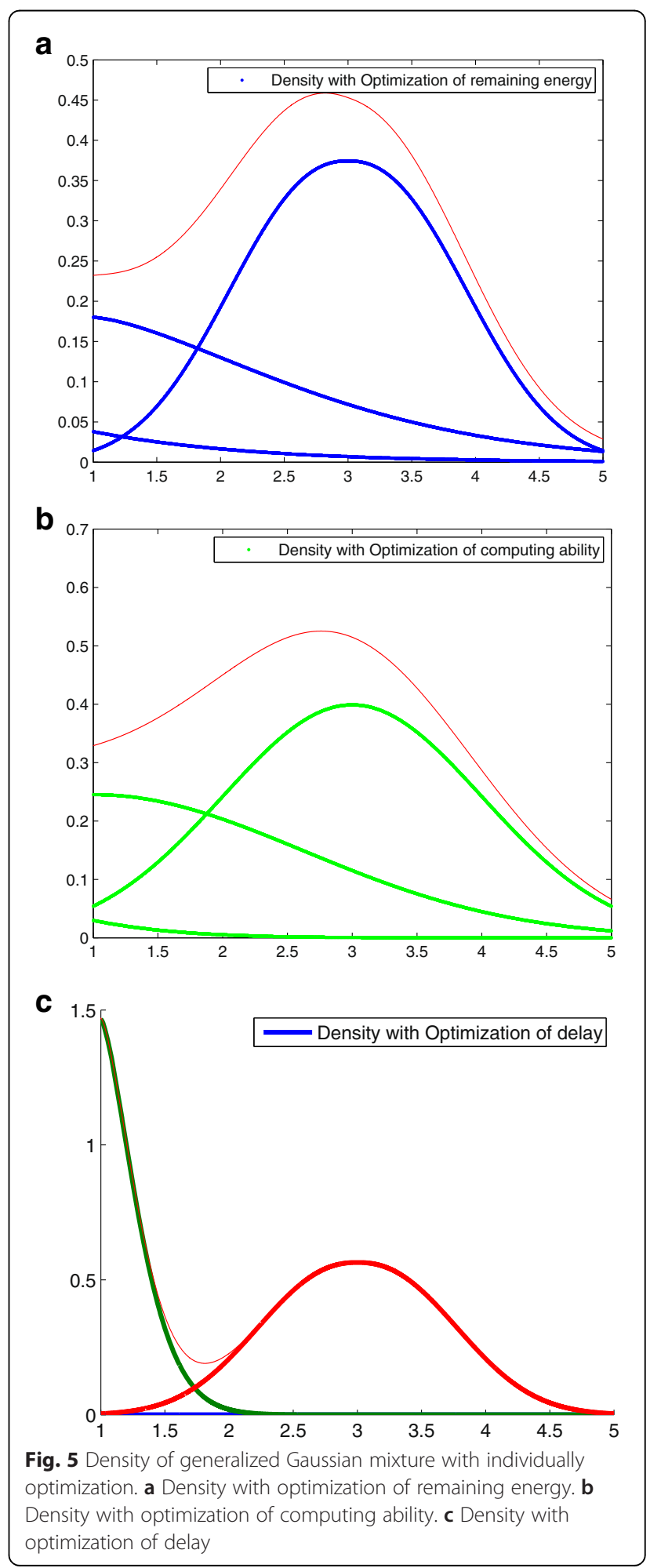


density function could maintain a reasonable trend because of the optimized balance weights.

Figure 6 shows the case of the generalized Gaussian mixed density function optimization based on the following three objectives with a balanced comprehensive optimization that indicates algorithm using the optimal weights $\mu$ can keep the characteristic distribution density function under conditions of integrated optimization.

Above all, the image processing algorithm based on generalized Gaussian mixture with optimization can be summarized as the following algorithm.

Input: Image data matrix $\mathrm{X}$, the real time information vector matrix $\Phi$.

Output: Image matrix Y

(1)According to the actual measurement, the matrix of Gaussian white noise with zero mean $[N, \cdots, N]^{T}$ would be set.
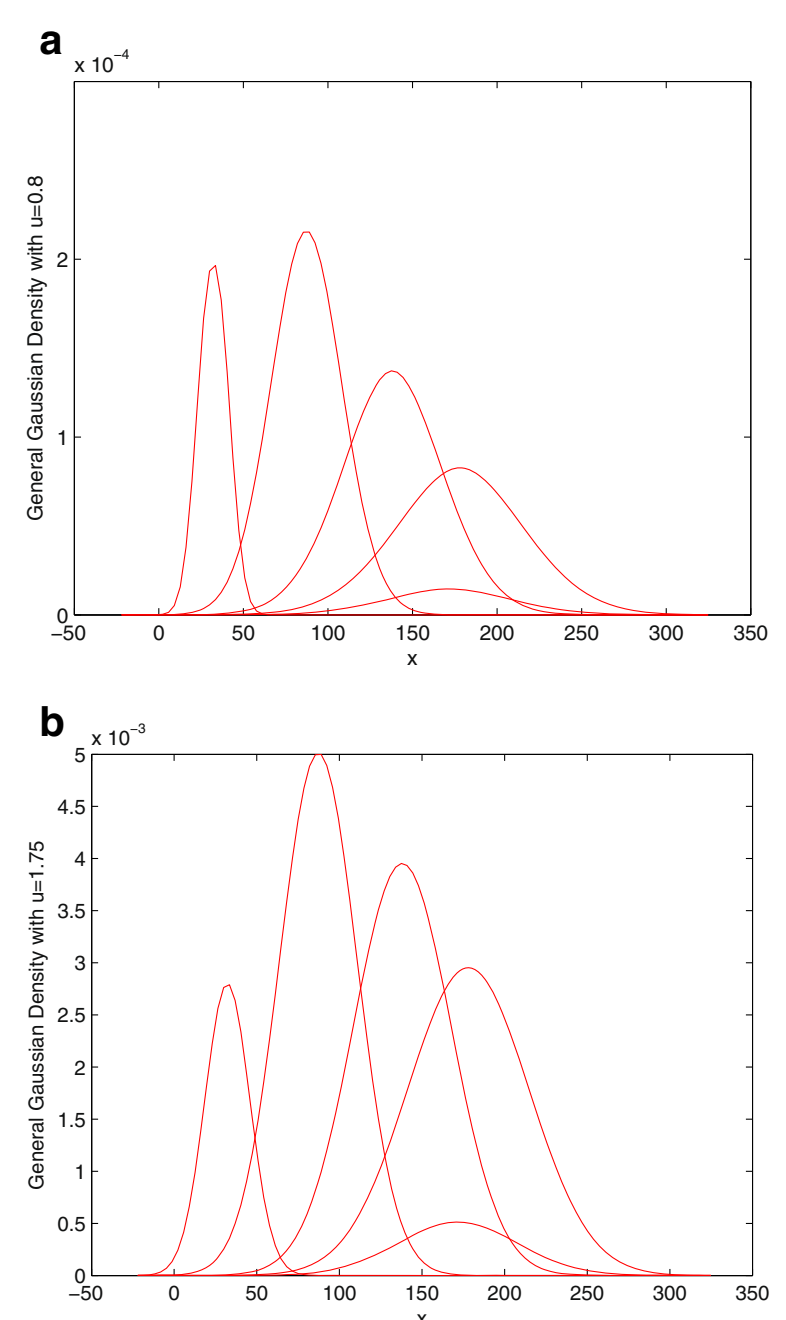

Fig. 6 Density of generalized Gaussian mixture with comprehensive optimization. $\mathbf{a} \mu=0.8$. $\mathbf{b} \mu=1.75$
(2) Numerical maximization function of the object optimization. The parameters of formula (4) are updated from 1 to $M$.

(3) According to the Generalized Gaussian mixture with comprehensive optimization, the matrix $\left[y_{1}, \ldots ., y_{M}\right]^{T}$ would be calculated for each image at sending sensor.

(4) Output the new image matrix Y.

In the above algorithm, we denote the two-dimensional signal matrix of image detection as Image matrix Y.

\section{Adaptive image processing and transmission scheme}

According to the analysis results of image transmission with opportunistic networking and image processing with generalized Gaussian mixture algorithm, the adaptive image processing and transmission scheme (GGMON) based on the combination of the above technology is proposed in this section.

Generalized Gaussian mixture algorithm based on opportunistic networking is shown as formula (5).

$$
p(\bar{X})=\sum_{l=1}^{M} \mu_{l} G\left(D_{l}, E_{R l}, A_{C l}\right) f(\bar{G})
$$

Here, let $M$ denote the mixed model number. $D_{l}$ represents the delay between the sending sensor and forwarding sensor. $E_{R l}$ represents the remaining energy of the identification 1 sensor. $A_{C l}$ represents the computing ability of the identification 1 sensor. Function $f(\bar{G})$ is used to select the relay sensors on basis of the directed graph $\bar{G}$. According to the principle of statistics, a weighted linear combination of some generalized Gauss probability density can approximate arbitrary distribution.

The proposed algorithm needs to pay attention to the following problems in the practical application.

(1)The image of relay sensors satisfies the following relationship in WSNs services, which is processed by generalized Gaussian mixture algorithm and transmitted with opportunistic networking.

$$
y=\left\{\begin{array}{cl}
x_{f(\bar{G})} \oplus N+\tau X & E_{R} \geq E_{R T} \& P_{\text {out }}=0 \\
x_{f(\bar{G})} \oplus N-\tau X & A_{C} \geq A_{C T} \& P_{\text {out }}=0 \\
x_{f(\bar{G})} \oplus N & D \geq D_{T} \& P_{\text {out }}=0 \\
0 & P_{\text {out }}=0
\end{array}\right.
$$

Here, $x_{f(\bar{G})}$ denotes the packet copy of image data at relay node. $\oplus$ denotes the XOR operation. $\tau$ represents the gray weight coefficient of image. We can initiate $\tau$ according to the random distribution analysis. $X$ is the 
interference signal variance in image transmission process. $E_{R T}, A_{C T}$, and $D_{T}$ are ,respectively, the threshold value of reaming energy, computing ability, and delay, which could be obtained by real measurement. $P_{\text {out }}$ is the outage probability.

(2)The end to end quality of service performance could be evaluated by the following metrics: average delay, packet error rate, and energy efficiency.

The energy efficiency $\eta$ of the proposed scheme could be obtained by formula (7).

$$
\eta=\frac{E_{s}+E_{f(\bar{G})}}{E_{\text {total }}}
$$

Here, $E_{s}$ is the energy consumption of sending sensor. $E_{\text {total }}$ is the total energy consumption of the WSNs. $E_{f(\bar{G})}$ is the energy consumption of relay sensors with opportunistic networking.

According to formula (8), the end to end average delay could be calculated.

$$
T_{a}=\frac{T_{s}+T_{f(\bar{G})}}{M}
$$

\section{Experiment results and evaluation}

In this section, we study, evaluate, and compare the QoS performance of the image processing scheme with opportunistic networking alone (IPONA) and the proposed GGMON in experiment 1 . On the other hand, the quality of encoded image at receiving sensor was evaluated by experiment 2 between image transmission with generalized Gaussian mixture algorithm alone (ITGGMA) and the proposed GGMON.

The results of experiment 1 are given by Fig. 7. From the three figures, we found that the QoS of GGMON is better than the IPONA obviously. The GGMON can maintain high reliability, real-time performance, and energy efficiency. These advantages are benefited from the opportunistic networking, which can obtain the gain of space, time, and antenna, and improve resource utilization such as energy, channel, etc.

The analysis result of experiment 2 is shown as Figs. 8 and 9. Experiment results show that the GGMON has better quality of image preservation than the ITGGMA approach. It is well known that the higher the peak signal to noise ratio the better the image quality. Although the performance of the two algorithms increases with the increase of the balanced weighting factor, the proposed algorithm is relatively slow decline. Especially, the peak signal to noise ratio and image contrast error tends to smooth
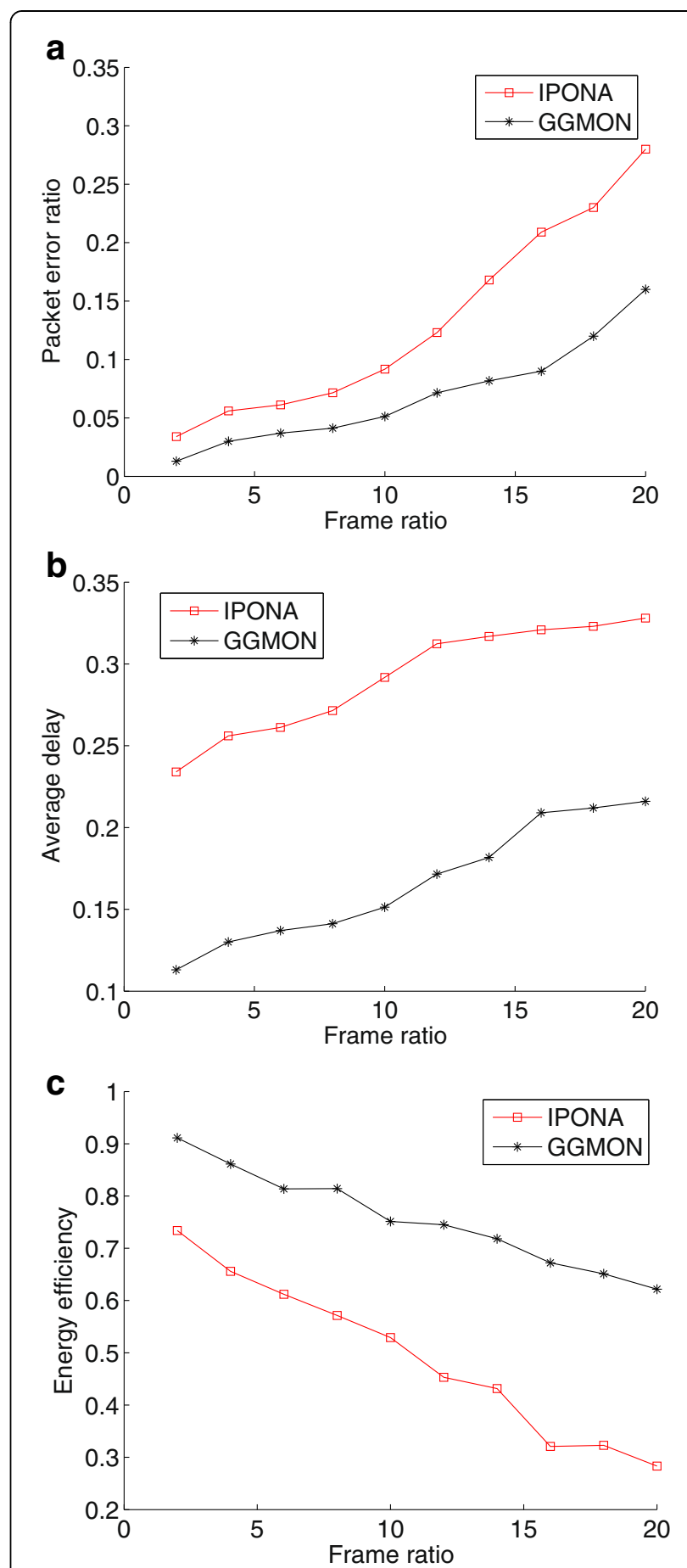

Fig. 7 QoS performance analysis. a Packet error ratio. b Average delay. c Energy efficiency

when the coefficient be larger. From Fig. 9, the proposed GGMON has better visual quality than the others. Experiment results show that the proposed method in our work can be applied to the image services in WSNs. The main reason is that we use the generalized Gaussian mixture algorithm with 


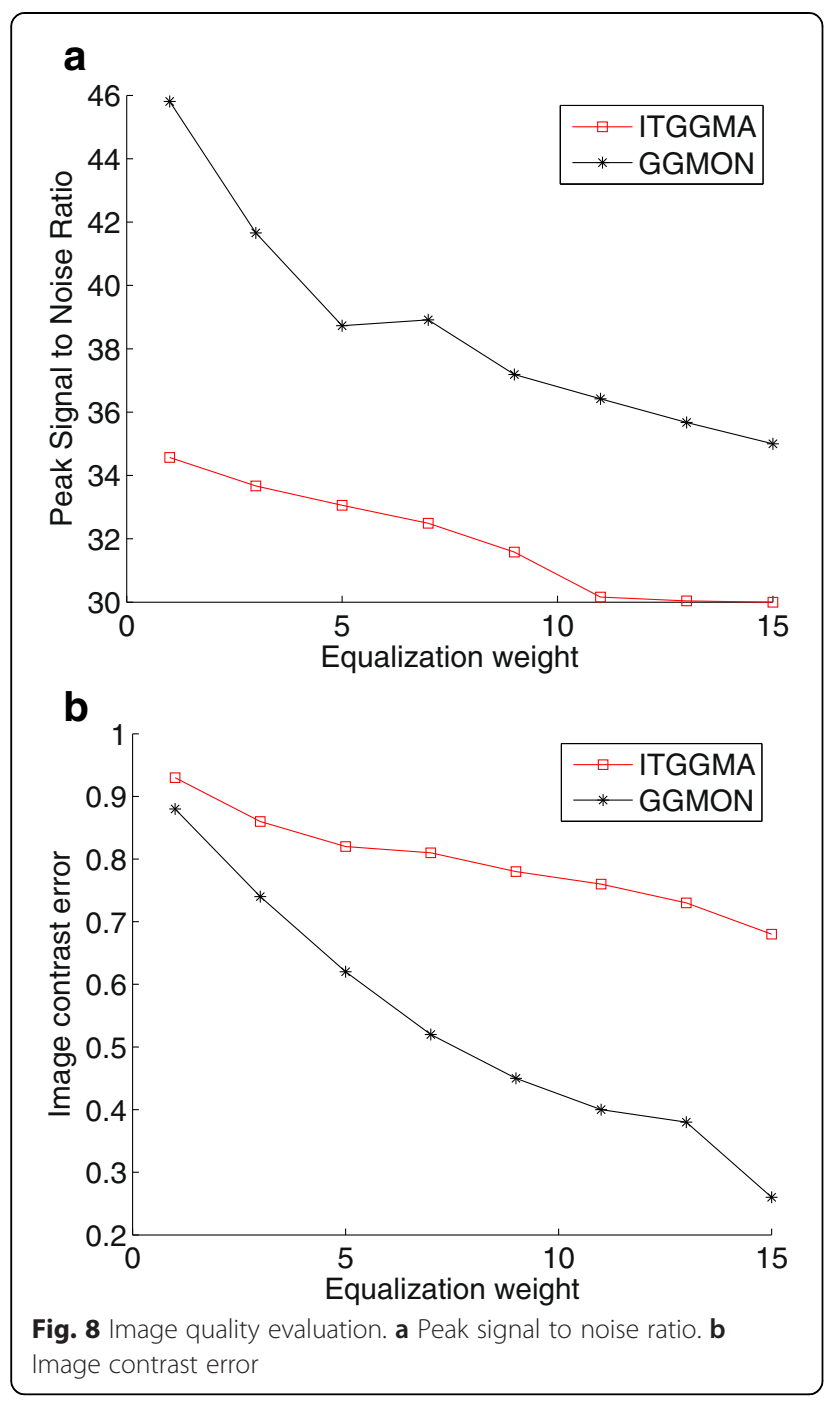

opportunistic networking before sending the image data in WSNs, which could compress the image data effectively and optimize system resources.

\section{Conclusions}

Image processing and transmission in WSNs has been extensively researched and developed in various application services, including traffic flow monitoring system, vehicle license plate recognition, etc. For gathering, processing, and transporting the image big data, we study the image transmission with opportunistic networking technology, which select the optimal relay sensors based on the real-time status including remaining energy and computing ability. Then, we research the image processing with generalized Gaussian mixture algorithm, which could compress the big image data effectively and eliminate the interference noise of image data caused in network transmission progress. In our work, we have proposed
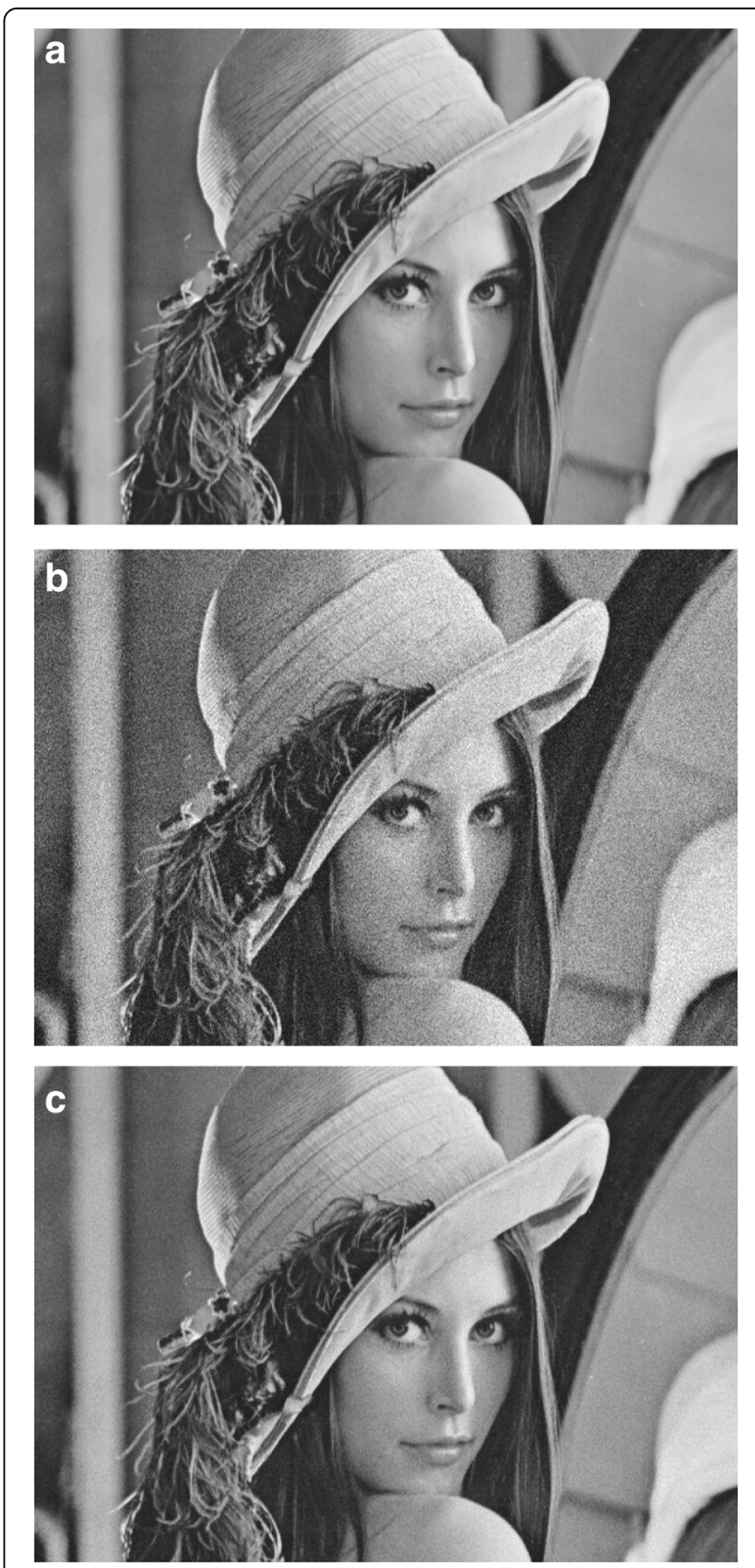

Fig. 9 Real image comparison. a Original image. b Image with ITGGMA. c Image with GGMON

the adaptive image processing and transmission scheme based on the combination of the above technology, called GGMON. It aims to improve the QoS performance of image transmission in WSNs while maintaining the high quality of image at receiving sensor. Experimental results on mathematical analysis and simulation show that the proposed scheme could obtain the better performance for image processing and transmission in WSNs. 


\section{Consent}

Written informed consent was obtained from the patient's guardian/parent/next of kin for the publication of this report and any accompanying images.

\section{Competing interests}

The authors declare that they have no competing interests.

\section{Acknowledgements}

This work was supported by National Natural Science Foundation of China (51305443), Natural Science Foundation of Jiangsu Province (bk20130184), Fundamental Research Funds for the Central Universities (2012QNA27), and A Project Funded by Priority Academic Program Development of Jiangsu Higher Education Institutions (PAPD).

Received: 10 July 2015 Accepted: 5 October 2015

\section{Published online: 13 October 2015}

\section{References}

1. E Menegatti, M Danieletto, M Mina, A Petto, A Bardellas S Zanconato et al., Autonomous discovery, localization and recognition of smart objects through WSN and image features. 2010 IEEE globecom Workshops (GC Wkshps), 2010, pp. 1653-1657

2. C Li Wern, A Li-Minn, S Kah Phooi, Survey of image compression algorithms in wireless sensor networks. Int Symposium Information Technol (ITSim) 4, 1-9 (2008)

3. CWH Ngau, L-M Ang, KP Seng, Low memory visual saliency architecture for data reduction in wireless sensor networks. IET Wireless Sensor Syst 2(2), 115-127 (2012)

4. V Lecuire, C Duran-Faundez, N Krommenacker, Energy-efficient image transmission in sensor networks. Int J Sensor Networks 4(1-2), 37-47 (2008)

5. T Sacha, D Bernhard, S Dominik, L Franck, WiFi-Opp: ad-hoc-less opportunistic networking. Proceedings of the 6th ACM workshop on Challenged networks (ACM New York, NY, USA, 2011), pp. 37-42

6. SA Muhammad Imran Razzak, AA Hussain, MS Minhas, Collaborative image compression in wireless sensor networks. Int J Computat Cognition 8(1), 24-29 (2010)

7. N Kimura, S Latifi, A survey on data compression in wireless sensor networks. Proceedings of 2005 International Conference on Information Technology: Coding and Computing (ITCC), vol. 2, 2005, pp. 8-13

8. Q Lu, W Luo, J Wang, B Chen, Low-complexity and energy efficient image compression scheme for wireless sensor networks. Comput. Netw. 52(13), 2594-2603 (2008)

9. ML Kaddachi, A Soudani, V Lecuire, K Torki, L Makkaoui, JM Moureaux. Low power hardware-based image compression solution for wireless camera sensor networks. Comp 10.1186/s13638-015-0458-3 Moureaux Low power hardware-based image compression solution for wireless camera sensor networks. Comp Standards Interfaces 34(1), 14-23 (2012)

10. G Nikolakopoulos, D Kandris, A Tzes, Adaptive compression of slowly varying images transmitted over wireless sensor networks. Sensors 10(8), 7170-7191 (2010)

11. SK Soni, N Chand, DP Singh, Reducing the data transmission in WSNs using time series prediction model. Proceedings of 2012 IEEE International Conference on Signal Processing, Computing and Control (ISPCC), 2012, pp. 1-5

12. K Irgan, C Ünsalan, S Baydere, Low-cost prioritization of image blocks in wireless sensor networks for border surveillance. J. Netw. Comput. Appl. 38, 54-64 (2014)

13. SM Aziz, DM Pham, Energy efficient image transmission in wireless multimedia sensor networks. IEEE Commun. Lett. 17(6), 1084-1087 (2013)

14. M Nasri, A Helali, H Sghaier, H Maaref. Adaptive image transfer for wireless sensor networks (WSNs). Proceedings of 2010 5th International Conference on Design and Technology of Integrated Systems in Nanoscale Era (DTIS), 2010, pp. 1-7

15. DG Costa, LA Guedes, Energy-efficient visual monitoring based on the sensing relevancies of source nodes for wireless image sensor networks. Proceedings of 2012 IEEE Sensors Applications Symposium (SAS), 2012, pp. 1-6

16. DM Pham, SM Aziz, Object extraction scheme and protocol for energy efficient image communication over wireless sensor networks. Comput. Netw. 57(15), 2949-2960 (2013)
17. X Liu, L Huang, J Li, Z Wang, D Zhao, M Zhu. Image transmission over ZigBee-based wireless sensor networks. Sens. Lett. 10(1-2), 205-212(8) (2012)

18. C Pham, V Lecuire, J-M Moureaux, Performances of multi-hops image transmissions on IEEE 802.15.4 wireless sensor networks for surveillance applications. Proceedings of 2013 IEEE 9th International Conference on Wireless and Mobile Computing, Networking and Communications (WiMob), 2013, pp. 477-484

19. L Pelusi, A Passarella, M Conti, Opportunistic networking: data forwarding in disconnected mobile ad hoc networks. IEEE Commun. Mag. 44(11), 134-141 (2006)

20. T Hossmann, G Nomikos, T Spyropoulos, F Legendre. Collection and analysis of multi-dimensional network data for opportunistic networking research. Comput. Commun. 35(13), 1613-1625 (2012)

21. J Gebert, R Fuchs, Probabilities for opportunistic networking in different scenarios, Future Network \& Mobile Summit (FutureNetw), 2012, pp. 1-8

22. M Conti, S Giordano, M May, A Passarella, From opportunistic networks to opportunistic computing. IEEE Commun. Mag. 48(9), 126-139 (2010)

23. H Wang, D Peng, W Wang, $H$ Sharif, $H$ Chen. Image transmissions with security enhancement based on region and path diversity in wireless sensor networks. IEEE Trans. Wirel. Commun. 8(2), 757-765 (2009)

24. EB Manhas Jr, G Brante, RD Souza, ME Pellenz. Energy-efficient cooperative image transmission over wireless sensor networks. Proceedings of 2012 IEEE Wireless Communications and Networking Conference (WCNC), 2012, pp. 2014-2019

25. MYS Uddin, H Wang, F Saremi, G Qi, T Abdelzaher, T Huang. PhotoNet: a similarity-aware picture delivery service for situation awareness. Proceedings of 2011 IEEE 32nd Real-Time Systems Symposium (RTSS), 2011, pp. 317-326

26. Z. Ren, S. Peng, H. Chen, J Fang, Q Chen. Epidemic routing based on adaptive compression of vectors: efficient low-delay routing for opportunistic networks based on adaptive compression of vectors. International Journal of Communication Systems (2013)

27. S. LV, J. Liu, A novel signal separation algorithm based on compressed sensing for wideband spectrum sensing in cognitive radio networks, International Journal of Communication Systems (2013)

28. L Wei, S Prasad, JE Fowler, Hyperspectral image classification using Gaussian mixture models and Markov random fields. IEEE Geosci. Remote Sens. Lett. 11(1), 153-157 (2014)

29. MS Allili, D Ziou, N Bouguila, S Boutemedjet. Image and video segmentation by combining unsupervised generalized Gaussian mixture modeling and feature selection. IEEE Trans Circuits Syst Video Technol 20(10), 1373-1377 (2010)

30. T Elguebaly, N Bouguila, Bayesian learning of finite generalized Gaussian mixture models on images. Signal Process. 91(4), 801-820 (2011)

\section{Submit your manuscript to a SpringerOpen ${ }^{\circ}$ journal and benefit from:}

- Convenient online submission

- Rigorous peer review

- Immediate publication on acceptance

- Open access: articles freely available online

- High visibility within the field

- Retaining the copyright to your article

Submit your next manuscript at $>$ springeropen.com 